\title{
Superacidity in Nafion/MOF Hybrid Membranes Retains Water at Low Humidity to Enhance Proton Conduction for Fuel Cells
}

\author{
Hasmukh A. Patel, ${ }^{*},{ }^{\dagger}{ }^{\S}$ Noramalina Mansor, ${ }^{\ddagger}$ Srinivas Gadipelli, ${ }^{\dagger}$ Dan J. L. Brett, ${ }^{\ddagger}$ and Zhengxiao Guo ${ }^{*}{ }^{\dagger}$ \\ ${ }^{\dagger}$ Department of Chemistry, University College London (UCL), London, WC1H 0AJ, United Kingdom \\ \#Electrochemical Innovation Lab, Department of Chemical Engineering, University College London (UCL), London WC1E 7JE, \\ United Kingdom
}

Supporting Information

ABSTRACT: A hybrid membrane of superacid sulfated $\mathrm{Zr}-\mathrm{MOF}$ (SZM) and Nafion shows much superior performance to Nafion, particularly for fuel cell operating under low humidity. The Brønsted acidic sites in SZM networks retain an ample amount of water which facilitated proton conduction under low humidity. The water retention properties of Nafion-SZM hybrid membranes with 1 wt \% loading of SZM increased at 35\% relative humidity and outperformed commercial unfilled Nafion membrane. The proton conductivity increases by $23 \%$ for Nafion-SZM hybrid compared to unfilled Nafion membrane. The Nafion-SZM membrane also shows higher performance stability at $35 \%$ relative humidity than Nafion, as confirmed by close monitoring of the change of open circuit voltage for $24 \mathrm{~h}$.

KEYWORDS: fuel cell, membrane, proton conduction, metal-organic frameworks, superacidity

F uel cells offer a highly efficient, clean, and low carbon technology for converting chemical to electrical energy, compared with heat engines. ${ }^{1,2}$ However, current proton exchange membrane fuel cells (PEMFCs) face technological challenges_catalyst poisoning and deterioration in proton conduction of the widely used Nafion electrolyte membrane at low humidity and limit the performance and efficiency of PEMFCs. ${ }^{3}$ Proton conductivity in PEMFCs is governed by comprehensive structural water molecules therein. It deteriorates under low humidity and/or high temperature, due to rapid loss of water molecules from the membrane. Alternatives, or modifications, to Nafion that can greatly improve proton conductivity at low humidity are therefore highly desirable.

Metal-organic frameworks (MOFs) have received much attention because of modular synthetic design, crystallinity, and useful properties in gas storage, selective separation, heterogeneous catalysis, charge carriers, and sensors. ${ }^{4,5}$ Recently, the study of proton conductivity in MOFs has gained momentum and it has been found that protons can travel through the coordination framework of MOFs or through carriers. ${ }^{6-10}$ However, the bulk phase and grain boundaries of MOFs restrict facile transfer of protons over long-range order, the proton conductivity of MOFs is limited. Self-assembly and hybridization strategies of MOFs architectures have been developed for practical applications, especially MOFs for device applications where processing and formulation are essential. ${ }^{11-13}$ Excellent proton carrier ability of MOFs can be realized if hybridized with polymeric matrices to improve distance conductivity. ${ }^{2}$ Water is essential for effective proton transfer in Nafion based PEMFCs. Such membranes show drastic decline in proton conduction at high temperature $(>80$ ${ }^{\circ} \mathrm{C}$ ) because the low relative humidity $(\mathrm{RH})$ at high temperature reduces substantially. The proton carrier mechanism at low RH through water can be facilitated if constituents of the membrane retain water at high temperature or low $\mathrm{RH}$. Indeed, water retention in the confined architecture of MOFs at high temperature provides a pathway to (i) enhance proton conduction, (ii) reduce poisoning of $\mathrm{Pt}$ in the anode, and (iii) provide a large operation window in commercial products. Exceptional water uptake properties of MOFs have been demonstrated, especially in a sulfated $\mathrm{Zr}-\mathrm{MOF}-808 .^{14,15}$ The integration of hygroscopic metal oxides, heteropolyacids, organic-inorganic hybrids and silicates- $\mathrm{SiO}_{2}, \mathrm{TiO}_{2}, \mathrm{ZrO}_{2}$, zeolites, etc., in Nafion have been studied. ${ }^{16-19}$ The electrostatically bonded structural water within the framework does not evaporate at high temperature and the insolubility in water restricts leaching of these materials from the membrane. The hydrolytically stable polymer/MOF hybrid composites have also proved good proton conductors under low humidity. ${ }^{20-22}$ A key advantage of MOFs over other metal oxide materials is that they can provide interconnected microporous networks to facilitate proton conduction, in addition to the confined water in hybrid membranes.

Received: September 26, 2016

Accepted: November 4, 2016

Published: November 4, 2016 

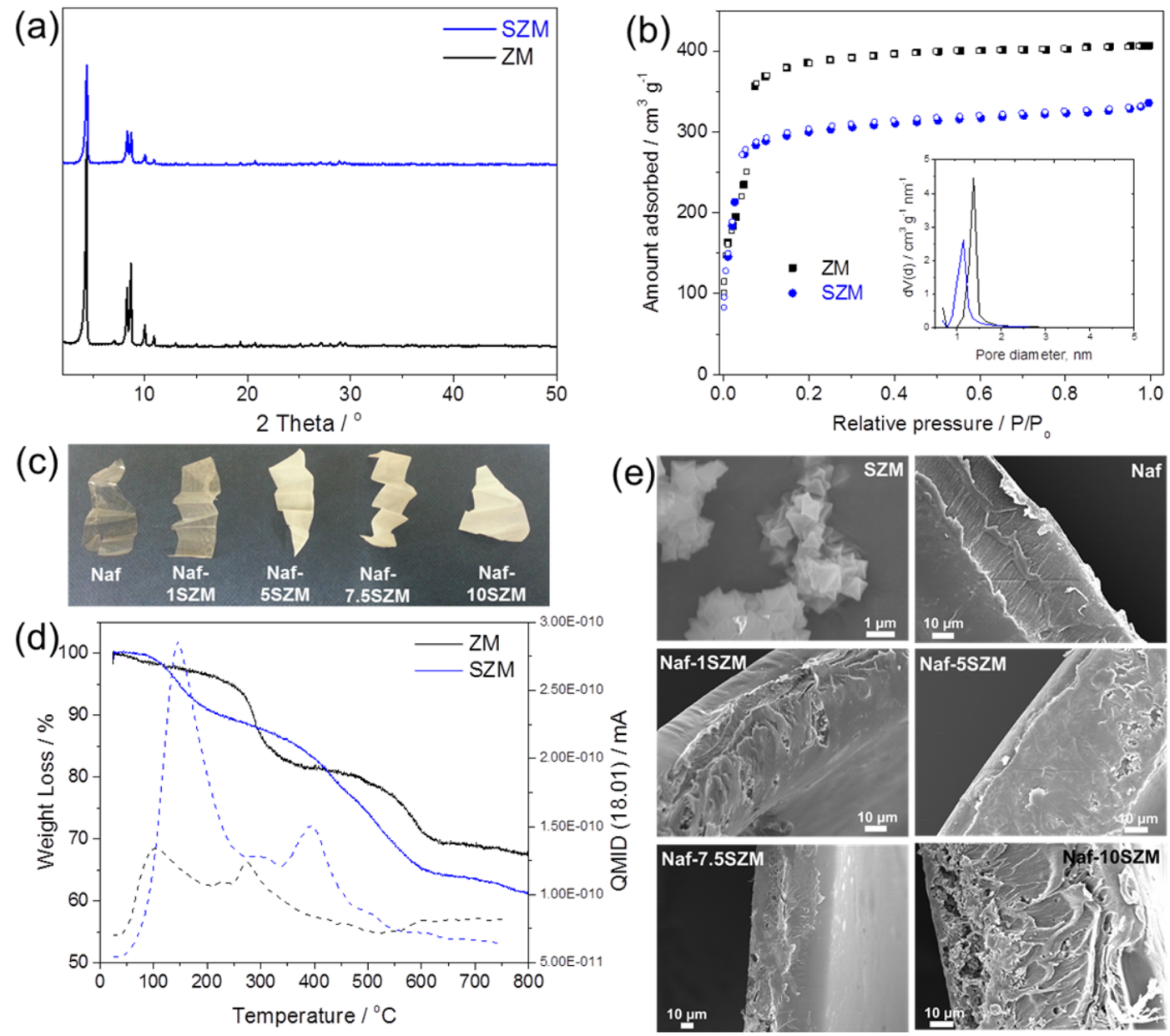

Figure 1. (a) Powder X-ray diffraction patterns of ZM and SZM. (b) $\mathrm{N}_{2}$ adsorption-desorption (filled and unfilled symbols represent adsorption and desorption respectively) isotherms at $77 \mathrm{~K}$ for ZM and SZM (inset: pore size distribution). (c) Pictorial illustration of squeezed Naf and NafSZM hybrid membranes with varied SZM loading $(1,5,7.5$, and $10 \mathrm{wt} \%$ ) showing stable membrane after activation (Supporting Information). (d) Thermogravimetric-mass spectrometry (TG-MS) spectra with quasi multiple ion detection (QMIS) of $\mathrm{H}_{2} \mathrm{O}$ for ZM and SZM show $\mathrm{H}_{2} \mathrm{O}$ molecules bonded at Brønsted acid sites of SZM evolved at higher temperature than ZM; and (e) SEM images of SZM and cross-section of Naf and Naf-SZM hybrid membranes.

Here, we report the water retention characteristics of a superacid $\mathrm{Zr}-\mathrm{MOF}$ compounded with Nafion under various concentrations to form membranes for use in PEMFCs, operating over a wide range of humidities that potentially offer a large working window and/or high temperature performance. The water molecules binding with superacid sites of sulfated $\mathrm{Zr}-\mathrm{MOF}$ at higher temperature are also monitored by thermogravimetric-mass spectrometry (TGMS). Although there have been several reports showing proton conduction in MOFs, these studies were limited to measurement of pelletized MOFs or single crystal MOFs, which could not be used as electrolyte membranes in fuel cells. This study demonstrates the utilization of MOFs for distance proton conductivity through hybrid membranes in a technologically relevant embodiment.

A microcrystalline $\mathrm{Zr}-\mathrm{MOF}-808$ ( $\mathrm{ZM})-$ $\mathrm{Zr}_{6} \mathrm{O}_{5}(\mathrm{OH})_{3}(\mathrm{BTC})_{2}(\mathrm{HCOO})_{5}\left(\mathrm{H}_{2} \mathrm{O}\right)_{2}$ and sulfated $\mathrm{Zr}-$ $\mathrm{MOF}-808$ (SZM) - $\mathrm{Zr}_{6} \mathrm{O}_{5}(\mathrm{OH})_{3}(\mathrm{BTC})_{2}\left(\mathrm{SO}_{4}\right)_{2.5}\left(\mathrm{H}_{2} \mathrm{O}\right)_{2.5}$ were synthesized according to the reported synthesis route, ${ }^{15}$ with slightly modified methodology (Supporting Information). Powder X-ray diffraction (PXRD) of ZM and SZM confirmed the well-crystalline phase formation and the excellent stability of SZM after sulfation in the acidic medium (Figure 1a). The stability of MOFs in water is also an important factor for use in PEMFCs as unstable MOFs or other hygroscopic materials can leach out over prolonged periods. The $\mathrm{N}_{2}$ adsorptiondesorption isotherms at $77 \mathrm{~K}$ elucidate type I behavior, which is typical of permanent microporosity (Figure 1b). The Brunauer-Emmett-Teller (BET) surface area was 1788 and $1263 \mathrm{~m}^{2} \mathrm{~g}^{-1}$ for $\mathrm{ZM}$ and SZM, respectively. The pore size distribution calculated from the BJH desorption curve, show the narrow pore size distribution with median pore size of $\mathrm{ZM}$ and SZM centered at 1.4 and $1.1 \mathrm{~nm}$, respectively. The solution casting of Nafion (Naf) and Naf-SZM hybrids were carried out using $N, N$-dimethylacetamide by dispersing SZM in Naf solution and casted in a petridish (Table S1). The hybrid membranes were obtained, as depicted in Figure 1c. Recently, the TG-MS study was employed to understand the mechanism of adsorbed water molecules on the proton conductivity in the UiO-66. ${ }^{23}$ In situ TG-MS was conducted for ZM and SZM to illuminate strong binding of water molecules at acidic sites within SZM frameworks compare to ZM (Figure 1d) at high temperature. Although ZM and SZM structures were thermally stable up to $300{ }^{\circ} \mathrm{C}$, SZM show $12 \%$ weight loss between $50-$ $280{ }^{\circ} \mathrm{C}$ which was attributed to the water evolution from water molecules attached with acidic sites and also from the 

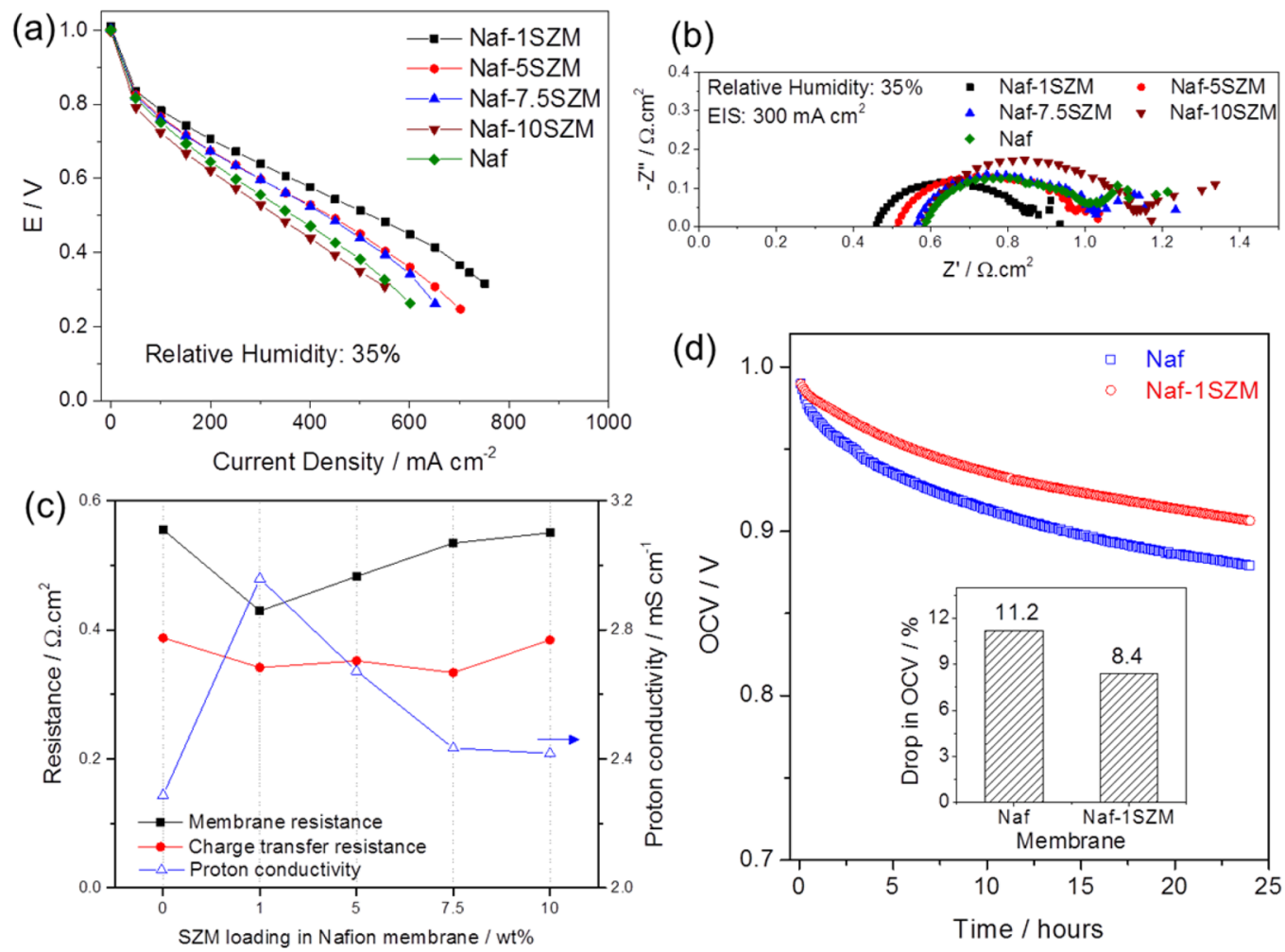

Figure 2. (a) Polarization $(I-V)$ curves of membrane electrode assemblies fabricated using Naf and Naf-SZM hybrid membranes, (b) Nyquist plots of Naf and Naf-SZM hybrid membranes obtained from electrochemical impedance spectroscopy (EIS) at $35 \% \mathrm{RH}$ and $80{ }^{\circ} \mathrm{C}$ fuel cell temperature, (c) membrane resistance, charge transfer resistance and proton conductivity with respect to SZM loading in Naf-SZM hybrid membranes, and (d) stability of Naf and Naf-1SZM membranes determined by holding open circuit voltage (OCV) at 35\% RH for $24 \mathrm{~h}$. Inset: drop in OCV after $24 \mathrm{~h}$ for Naf and Naf-1SZM membranes.

dehydoxylation of frameworks, ${ }^{24}$ as confirmed by quasi multiple ion detection (QMID) of water molecules. ZM shows evolution of water (6 wt \%) over the temperature range of 50-280 ${ }^{\circ} \mathrm{C}$. The QMID peak maxima for ZM and SZM was at 105 and $148{ }^{\circ} \mathrm{C}$ respectively, which confirmed the strong binding of water molecules with acidic sites for SZM, compared to ZM. Therefore, the superacidic sites and the highly microporous networks in SZM make it a promising proton conducting material for hybrid membranes at relatively low humidity and/or high temperature for PEMFCs.

The SEM image of SZM indicates the morphology of octahedral microcrystals (Figure 1e) was unaltered after sulfation of ZM (Figure S1) and the size of microcrystals were 200-900 $\mathrm{nm}$. The SEM cross-section images of NafSZM membranes with varied concentration of SZM $(1,5,7.5$, and $10 \mathrm{wt} \%$ ) show well-distributed SZM in the Naf matrix. However, the formation of pinholes and cracks were evidently visible above 5 wt \% loading, because of high concentration and large-sized microcrystals of SZM. Although the hybrid membranes were physically stable, microstructural distortion occurred at high SZM loading. The compatibility of SZM with Naf matrix also decreased at high concentration, as seen from SEM images (Figure S2). The optimum concentration of SZM was found to be 1 wt \% in Naf-SZM hybrids which show no pinholes and cracks. The Naf-SZM hybrid membranes showed excellent thermal stability up to $300{ }^{\circ} \mathrm{C}$ (Figure S3). The structural stability of SZM upon hybridization with Naf was confirmed by XRD and FT-IR. The XRD pattern clearly show major reflections from SZM at low angles in the Naf-SZM hybrids with 5, 7.5, and 10 wt \% SZM, though the Naf-1SZM does not show visible reflections due to the very low level of SZM in the membrane (Figure S4). Other reflections of SZM are overlapped by Naf matrix. The FT-IR spectrum of the NafSZM hybrids show characteristic vibrations from SZM and further confirms that the structure has not deteriorated upon mixing with Naf (Figure S5).

Additionally, the water uptake (WU) and ion-exchange capacity (IEC) of hybrid membranes were measured to validate effect of water retention properties. Comparison of the WU of SZM, Naf, and Naf-SZM hybrid membranes at different RHs shows that the Naf-SZM hybrid membrane constantly show higher WU than the Naf at different RHs (Figure S6). The results further support our argument that there exists higher water retention in the Naf-SZM hybrid than in Naf. The IEC was maintained upon addition of SZM in Nafion, around 0.85-

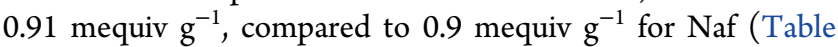
S2). Liang et al. ${ }^{21}$ demonstrated synthesis of chiral layered two-dimensional MOFs with intrinsic proton carriers and combined it with polyvinylpyrrolidone (PVP). The MOFloaded PVP hybrids showed enhancement in proton conductivity, higher than that of MOF and PVP at 53\% $\mathrm{RH}$ and 298 K. A mixed-matrix membrane prepared from sulfonated polymer and Fe-MIL-101- $\mathrm{NH}_{2}$ offered cooperative proton conductivity from sulfonimide moieties of polymeric matrix and water bound to the open metal site of $\mathrm{Fe}(\mathrm{III}) .^{22}$ These studies reflected the need of developing MOF-based hybrid systems which can function at very low humidity as well as exhibit functioning of polymer/MOF electrolyte membrane in membrane electrode assemblies of fuel cell. 
The fuel cell polarization profiles of membrane electrode assemblies (MEAs) prepared of Naf and Naf-SZM hybrid membranes obtained at $35 \% \mathrm{RH}$ and $80{ }^{\circ} \mathrm{C}$ are shown in Figure 2a. The performance of Naf-1SZM, Naf-5SZM, and $\mathrm{Naf}-7.5 \mathrm{SZM}$ are better than the commercial Naf, which can be attributed to the ability of SZM to retain water. The Nyquist plots of Naf and Naf-SZM obtained from electrochemical impedance measurements at $300 \mathrm{~mA} \mathrm{~cm}{ }^{-2}$ and $35 \% \mathrm{RH}$ are shown in Figure $2 \mathrm{~b}$. The $Z^{\prime}$ axis intercept of the arc at high frequency corresponds to Ohmic resistance of the cell, which includes the proton transport resistance in the membrane. The results from Nyquist plot equivalent circuit fittings and proton conductivity are shown in Figure $2 \mathrm{c}$ and Table S2. Consistent with the polarization curves, the incorporation of SZM in Naf increases the proton conductivity of Naf-SZM hybrid membranes.

As all the membranes have similar thickness, the increase in proton conductivity strongly suggest that the incorporation of SZM materials improves the ability of the membrane to retain water. However, increasing the SZM loading higher than $1 \mathrm{wt}$ $\%$ does not further increase the proton conductivity, which could be due to the formation of pinholes and cracks at high SZM loadings (Figure 1e). The charge transfer resistance is expected to be similar since the same Pt electrodes are used for all MEAs but the Nyquist plot and equivalent circuit fitting show that charge transfer resistance increases with Ohmic resistance. It is believed that proton conductivity also has an effect on charge transfer kinetics in the electrodes because of diffusional limitations of protons, which are necessary to complete electrochemical reactions. ${ }^{25}$ Nevertheless the variations in charge transfer resistances between Naf and Naf-SZM are insignificant compared to the change in membrane resistance and proton conductivity, as shown in Figure 2c. The long-term performance of composite membranes is indeed one of the requirements for PEMFCs. Therefore, the open circuit voltage was measured by holding the membranes at $35 \%$ $\mathrm{RH}$ for $24 \mathrm{~h}$. Since Naf-1SZM is the best performing membrane, OCV hold test was carried out to study its durability. It was reported that gas permeability of the membrane is the main factor affecting degradation during OCV hold. ${ }^{26,27}$ Hydrogen crossover through the membrane reacts with oxygen on the platinum catalyst electrode, causing mixed potential which reduces the OCV. In addition, the reaction also forms radicals and hydrogen peroxide, which are responsible for membrane degradation. Figure $2 \mathrm{~d}$ presents the OCV hold test results and corresponding percentage drop in OCV with respect to the initial OCV. Naf-1SZM composite membranes show better durability than Naf with OCV drop of $8.4 \%$, compared to $11 \%$ for $\mathrm{Naf}$.

The proton conductivity in solid polymer electrolyte materials is categorized in two principle mechanisms: vehicle and Grotthuss. ${ }^{28-31}$ The hydrophilic interface between Naf and SZM facilitate proton conduction in Naf-SZM hybrid. Furthermore, proton percolation pathways at the interfaces reduces the amount of water to maintain good proton conduction. $^{31}$ An imidazole functionality was found to be excellent proton conductor through the vehicle mechanism, while structural water molecules promote the Grotthuss mechanism in MOF. ${ }^{32,33}$ The hydrogen bonded water networks at highly acidic sulfated functionalities in SZM provide proton conduction through Grotthuss mechanism for the Naf-SZM hybrid membranes. A similar characteristics is recently observed for Brønsted acid sites $\left(-\mathrm{SO}_{3} \mathrm{H}\right)$ functionalized $\mathrm{UiO}-66$ on the strut through post-synthesis modification which facilitated formation of hydrophilic domains in the frameworks. ${ }^{8}$ For comparison, the polarization curves and impedance spectra at high relative humidity $(60,80$, and $98 \%)$ are shown in Figures S7 and S8. All the MEAs show almost identical performance at low current density. The results indicate that the effect of SZM in Naf is most prominent under low humidity conditions (35\% $\mathrm{RH}$ ) where these hybrid membranes are outperforming unfilled Naf, because of strongly bonded water molecules at Brønsted acidic sites of SZM which contributes to proton conduction enhancement.

In summary, we have demonstrated a strategy for improving long-range-order proton conductivity through utilization of superacidic sites of SZM by means of Naf-SZM hybrid membranes. A TG-MS study suggests that intrinsic water molecules in SZM networks strongly bond with acidic moiety and evolve at high temperature, which leads to excellent proton conductivity and fuel cell performance at $35 \% \mathrm{RH}$. The WU and IEC study of Naf-SZM show excellent water retention at different RHs and no deterioration of IEC of Naf upon hybridization. The voltage at $500 \mathrm{~mA} \mathrm{~cm}^{-2}$ is improved by $34 \%$ and Ohmic resistance decreases upon blending of 1 wt \% SZM in a Naf matrix. The Ohmic resistance decreases upon blending of 1 wt \% SZM in a Naf matrix which result in 23\% increase in proton conductivity. Because the crystal sizes of SZM is in the range of 200-900 nm, microstructural distortion of the membrane occurred at high SZM loadings. The long-term durability test of Naf-1SZM show lower percentage drop in OCV compare to Naf. The study paves the way for further development of high-performance fuel cells that can be operated over a large operation window even under low humidity and/or high temperature, to avoid catalysis poisoning and maintain high proton conductivity.

\section{ASSOCIATED CONTENT}

\section{Supporting Information}

The Supporting Information is available free of charge on the ACS Publications website at DOI: 10.1021/acsami.6b12240.

Synthesis procedures, TGA, TG-MS, and SEM images of hybrid membranes, characterization and fuel cell testing details, and fuel cell performance (polarization and Nyquist plots) of hybrid membranes at high relative humidity (PDF)

\section{AUTHOR INFORMATION}

\section{Corresponding Authors}

*E-mail: hasmukhpatel@aol.in.

*E-mail: z.x.guo@ucl.ac.uk.

\section{Present Address}

${ }^{\S}$ H.A.P. is currently at Department of Chemistry, Northwestern University, 2145 Sheridan Road, Evanston, IL 60208-3113, USA.

\section{Notes}

The authors declare no competing financial interest.

\section{ACKNOWLEDGMENTS}

We thank Steve Firth for assistance with SEM, Martin Vickers for assistance with PXRD, and Quentin Meyer for assistance with UCL-FRA. The project involves support from the UK EPSRC under Grant EP/K002252/1, EP/L018330/1, EP/ $\mathrm{K} 021192 / 1 ; \mathrm{EP} / \mathrm{I037024/1;} \mathrm{EP} / \mathrm{M} 009394 / 1$ and EP/ M014371/1. 


\section{REFERENCES}

(1) Jiang, J.; Yaghi, O. M. Brønsted Acidity in Metal-Organic Frameworks. Chem. Rev. 2015, 115, 6966-6997.

(2) Ramaswamy, P.; Wong, N. E.; Shimizu, G. K. H. MOFs as Proton Conductors - Challenges and Opportunities. Chem. Soc. Rev. 2014, 43, 5913-5932.

(3) Giffin, G. A.; Haugen, G. M.; Hamrock, S. J.; Di Noto, V. Interplay between Structure and Relaxations in Perfluorosulfonic Acid Proton. J. Am. Chem. Soc. 2013, 135, 822-834.

(4) Sumida, K.; Rogow, D. L.; Mason, J. A.; McDonald, T. M.; Bloch, E. D.; Herm, Z. R.; Bae, T. H.; Long, J. R. Carbon Dioxide Capture in Metal-Organic Frameworks. Chem. Rev. 2012, 112, 724-781.

(5) Yoon, M.; Suh, K.; Natarajan, S.; Kim, K. Proton Conduction in Metal-Organic Frameworks and Related Modularly Built Porous Solids. Angew. Chem., Int. Ed. 2013, 52, 2688-2700.

(6) Bureekaew, S.; Horike, S.; Higuchi, M.; Mizuno, M.; Kawamura, T.; Tanaka, D.; Yanai, N.; Kitagawa, S. One-dimensional Imidazole Aggregate in Aluminium Porous Coordination Polymers with High Proton Conductivity. Nat. Mater. 2009, 8, 831-836.

(7) Hurd, J. A.; Vaidhyanathan, R.; Thangadurai, V.; Ratcliffe, C. I.; Moudrakovski, I. L.; Shimizu, G. K. H. Anhydrous Proton Conduction at $150{ }^{\circ} \mathrm{C}$ in a crystalline metal-organic framework. Nat. Chem. 2009, $1,705-710$.

(8) Phang, W. J.; Jo, H.; Lee, W. R.; Song, J. H.; Yoo, K.; Kim, B.; Hong, C. S. Superprotonic Conductivity of a UiO-66 Framework Functionalized with Sulfonic Acid Groups by Facile Postsynthetic Oxidation. Angew. Chem., Int. Ed. 2015, 54, 5142-5146.

(9) Ramaswamy, P.; Wong, N. E.; Gelfand, B. S.; Shimizu, G. K. H. A Water Stable Magnesium MOF That Conducts Protons over $10^{-2} \mathrm{~S}$ $\mathrm{cm}^{-1}$. J. Am. Chem. Soc. 2015, 137, 7640-7643.

(10) Taylor, J. M.; Dekura, S.; Ikeda, R.; Kitagawa, H. Defect Control to Enhance Proton Conductivity in a Metal-Organic Framework. Chem. Mater. 2015, 27, 2286-2289.

(11) Denny, M. S.; Cohen, S. M. In Situ Modification of MetalOrganic Frameworks in Mixed-Matrix Membranes. Angew. Chem., Int. Ed. 2015, 54, 9029-9032.

(12) Panda, T.; Kundu, T.; Banerjee, R. Self-assembled One Dimensional Functionalized Metal-Organic Nanotubes (MONTs) for Proton Conduction. Chem. Commun. 2012, 48, 5464-5466.

(13) Xu, G.; Otsubo, K.; Yamada, T.; Sakaida, S.; Kitagawa, H. Superprotonic Conductivity in a Highly Oriented Crystalline MetalOrganic Framework Nanofilm. J. Am. Chem. Soc. 2013, 135, 74387441.

(14) Furukawa, H.; Gandara, F.; Zhang, Y. B.; Jiang, J. C.; Queen, W. L.; Hudson, M. R.; Yaghi, O. M. Water Adsorption in Porous MetalOrganic Frameworks and Related Materials. J. Am. Chem. Soc. 2014, 136, 4369-4381.

(15) Jiang, J. C.; Gandara, F.; Zhang, Y. B.; Na, K.; Yaghi, O. M.; Klemperer, W. G. Superacidity in Sulfated Metal-Organic Framework808. J. Am. Chem. Soc. 2014, 136, 12844-12847.

(16) Adjemian, K. T.; Dominey, R.; Krishnan, L.; Ota, H.; Majsztrik, P.; Zhang, T.; Mann, J.; Kirby, B.; Gatto, L.; Velo-Simpson, M.; Leahy, J.; Srinivasan, S.; Benziger, J. B.; Bocarsly, A. B. Function and Characterization of Metal Oxide-Naflon Composite Membranes for Elevated-temperature $\mathrm{H}_{2} / \mathrm{O}_{2}$ PEM Fuel Cells. Chem. Mater. 2006, 18, 2238-2248.

(17) D’Epifanio, A.; Navarra, M. A.; Weise, F. C.; Mecheri, B.; Farrington, J.; Licoccia, S.; Greenbaum, S. Composite Nafion/Sulfated Zirconia Membranes: Effect of the Filler Surface Properties on Proton Transport Characteristics. Chem. Mater. 2010, 22, 813-821.

(18) Di Noto, V.; Bettiol, M.; Bassetto, F.; Boaretto, N.; Negro, E.; Lavina, S.; Bertasi, F. Hybrid Inorganic-organic Nanocomposite Polymer Electrolytes Based on Nafion and Fluorinated $\mathrm{TiO}_{2}$ for PEMFCs. Int. J. Hydrogen Energy 2012, 37, 6169-6181.

(19) Di Noto, V.; Piga, M.; Negro, E.; Giffin, G. A.; Polizzi, S.; Zawodzinski, T. A. New Nanocomposite Proton Conducting Membranes Based on a Core-Shell Banofiller for Low Relative Humidity Fuel Cells. RSC Adv. 2013, 3, 18960-18969.
(20) Li, Z.; He, G. W.; Zhang, B.; Cao, Y.; Wu, H.; Jiang, Z. Y.; Tiantian, Z. Enhanced Proton Conductivity of Nafion Hybrid Membrane under Different Humidities by Incorporating MetalOrganic Frameworks With High Phytic Acid Loading. ACS Appl. Mater. Interfaces 2014, 6, 9799-9807.

(21) Liang, X. Q.; Zhang, F.; Feng, W.; Zou, X. Q.; Zhao, C. J.; Na, H.; Liu, C.; Sun, F. X.; Zhu, G. S. From metal-organic framework (MOF) to MOF-Polymer Composite Membrane: Enhancement of Low-Humidity Proton Conductivity. Chem. Sci. 2013, 4, 983-992.

(22) Wu, B.; Lin, X. C.; Ge, L.; Wu, L.; Xu, T. W. A Novel Route for Preparing Highly Proton Conductive Membrane Materials with MetalOrganic Frameworks. Chem. Commun. 2013, 49, 143-145.

(23) Yang, F.; Huang, H.; Wang, X.; Li, F.; Gong, Y.; Zhong, C.; Li, J. R. Proton Conductivities in Functionalized UiO-66: Tuned Properties, Thermogravimetry Mass, and Molecular Simulation Analyses. Cryst. Growth Des. 2015, 15, 5827-5833.

(24) Cavka, J. H.; Jakobsen, S.; Olsbye, U.; Guillou, N.; Lamberti, C.; Bordiga, S.; Lillerud, K. P. A New Zirconium Inorganic Building Brick Forming Metal Organic Frameworks with Exceptional Stability. J. Am. Chem. Soc. 2008, 130, 13850-13851.

(25) Hink, S.; Wagner, N.; Bessler, W. G.; Roduner, E. Impedance Spectroscopic Investigation of Proton Conductivity in Nafion Using Transient Electrochemical Atomic Force Microscopy (AFM). Membranes 2012, 2, 237-252.

(26) Ohma, A.; Suga, S.; Yamamoto, S.; Shinohara, K. Membrane Degradation Behavior during Open-Circuit Voltage Hold Test. J. Electrochem. Soc. 2007, 154, B757-B760.

(27) Ohma, A.; Yamamoto, S.; Shinohara, K. Analysis of Membrane Degradation Behavior During OCV Hold Test. ECS Trans. 2007, 11, $1181-1192$

(28) Agmon, N. The Grotthuss Mechanism. Chem. Phys. Lett. 1995, 244, 456-462.

(29) Di Noto, V.; Boaretto, N.; Negro, E.; Giffin, G. A.; Lavina, S.; Polizzi, S. Inorganic-Organic Membranes Based on Nafion, $\left[\left(\mathrm{ZrO}_{2}\right)\right.$. $\left.\left(\mathrm{HfO}_{2}\right)_{0.25}\right]$ and $\left[\left(\mathrm{SiO}_{2}\right) \cdot\left(\mathrm{HfO}_{2}\right)_{0.28}\right]$. Part I: Synthesis, Thermal Stability and Performance in a Single PEMFC. Int. J. Hydrogen Energy 2012, 37, 6199-62149.

(30) Di Noto, V.; Boaretto, N.; Negro, E.; Pace, G. New InorganicOrganic Proton Conducting Membranes Based on Nafion and Hydrophobic Fluoroalkylated Silica Nanoparticles. J. Power Sources 2010, 195, 7734-7742.

(31) Di Noto, V.; Piga, M.; Giffin, G. A.; Vezzu, K.; Zawodzinski, T. A. Interplay between Mechanical, Electrical, and Thermal Relaxations in Nanocomposite Proton Conducting Membranes Based on Nafion and a $\left[\left(\mathrm{ZrO}_{2}\right) \cdot\left(\mathrm{Ta}_{2} \mathrm{O}_{5}\right)_{0.119}\right]$ Core-Shell Nanofiller. J. Am. Chem. Soc. 2012, 134, 19099-19107.

(32) Horike, S.; Umeyama, D.; Inukai, M.; Itakura, T.; Kitagawa, S. Coordination-Network-Based Ionic Plastic Crystal for Anhydrous Proton Conductivity. J. Am. Chem. Soc. 2012, 134, 7612-7615.

(33) Jeong, N. C.; Samanta, B.; Lee, C. Y.; Farha, O. K.; Hupp, J. T. Coordination-Chemistry Control of Proton Conductivity in the Iconic Metal-Organic Framework Material HKUST-1. J. Am. Chem. Soc. 2012, 134, 51-54. 